this study was to assess the effect of multitasking on cognitive load when learning a common trauma skill. Methods: Sixteen students who had no previous experience with the one-handed square knot were randomly assigned to one of the two groups: practice and non-practice groups. A pre-recorded instructional video of a one-hand knot tying was presented to all participants. Next, the practice group completed a single session of 10 trials of the one-hand knot tying using a benchtop simulator, while the non-practice group did not. All returned a week later for a transfer task on a different simulator. On trials 1, 4, 7,10 and during the transfer performance the participants performed under dual task conditions, where they were asked to focus on the knot-tying task, but also to react as fast as possible to the illumination of an incandescent light bulb by pressing a foot pedal. Subjective ratings of mental effort, and reaction time to the visual stimulus were used as indices of cognitive load. Results: A repeated measure ANOVA showed a significant effect of dual task on subjective measure of mental effort $\left(F_{(4,28)}=10.35, p=.001, \omega=.60\right)$ and reaction time $\left(F_{(4,28)}=\right.$ $7.93, p=.001, \omega=.53$ ), with plots indicating cognitive load plateaued by the $7^{\text {th }}$ trial. Conclusion: These findings highlights the number of trials necessary to attain a level of proficiency in a basic trauma skill such as the one-hand knot tying, ease the level of cognitive load and possibly enhance transfer to more challenging tasks.

Keywords: dual-task, trauma skills, cognitive load

\section{P005 \\ Pre-hospital dexamethasone administration in children with croup: the Edmonton experience}

S. Ali, MDCM, A. Moodley, MD, A. Bhattacharjee, BSc, K. Lobay, DMD, MD, E. Chang, MD, A. Kabaroff, MD, D. Allain, MD; University of Alberta, Edmonton, $\mathrm{AB}$

Introduction: Croup is one of the most common childhood respiratory illnesses, affecting more than 80,000 Canadian children per year. Early dexamethasone administration in croup can reduce admission rates and length of stay (LOS), as well as return visits to the emergency department (ED). Pre-hospital emergency medical services (EMS) teams in Edmonton administer dexamethasone to children with croup. The objectives of this study were to (a) assess the clinical impact of prehospital administration of dexamethasone to children with croup and (b) compare clinical outcomes of these patients to those who did not receive their first dose of dexamethasone via the EMS providers. Methods: This study was a retrospective medical record review that included children between 6 months and 6 years of age who were brought via EMS to the Stollery Children's Hospital ED with a final diagnosis of croup, between January 1st 2010 and December 31 2012. Data were collected regarding pre-hospital presentation and management, ED presentation and management, ED LOS and final disposition, and patient demographics. Results: 188 patients were enrolled, $35.1 \%$ (66/188) of whom received a pre-hospital diagnosis of croup. The mean age of the participants was 32.96 months ( $S D \pm 17.18$ ). Overall, $10.6 \%$ patients (20/188) were given dexamethasone in the pre-hospital setting, while $30.3 \%$ patients $(57 / 188)$ were given nebulized epinephrine by EMS. Out of the 66 patients with a pre-hospital diagnosis of croup, $10.6 \%$ $(\mathrm{n}=7)$ were given dexamethasone by EMS. In the ED, dexamethasone was administered to $88.3 \%$ of patients $(166 / 188)$ while $56 / 188$ participants $(29.8 \%)$ received nebulized epinephrine. There was no statistically significant difference in ED LOS stay between those who received pre-hospital dexamethasone (2.6 hours, $\mathrm{SD} \pm 1.6, \mathrm{n}=18$ ) and those who did not (3.3 hours, $\mathrm{SD} \pm 2.7, \mathrm{n}=159$ ). The number of in-hospital epinephrine doses per patient was influenced by the administration of pre-hospital dexamethasone $(\mathrm{p}=0.010)$. Conclusion: Pre-hospital administration of dexamethasone likely influences the severity and short-term persistence of croup symptoms, as evidenced by less epinephrine use in the ED. Contrary to current EMS guidelines, very few patients with a pre-hospital diagnosis of croup received dexamethasone by EMS personnel. This likely represents a missed opportunity to decrease the severity of the patients' disease.

Keywords: emergency medical services (EMS), croup, dexamethasone

\section{P006}

A qualitative study of the language of satisfaction for children in the emergency department

S. Ali, MDCM, T. McGrath, MD, N. Dow, BA, S. Aziz, BSc, M. Pilarski, MD, A.L. Drendel, DO, MS; University of Alberta, Edmonton, $\mathrm{AB}$

Introduction: Measures of satisfaction are essential to understanding patient experience, and pain management. Currently, there are no validated tools to quantify children's satisfaction. To develop such a tool, we must first understand which words children use to communicate satisfaction. Our objectives were to (A) to identify the words commonly used by children of different ages to communicate satisfaction, in general, and in the context of pain management, and (B) to determine if this vocabulary is similar to that used by their caregiver. Methods: A qualitative study of 105 children-parent pairs, aged 3-16 years, who were evaluated at a pediatric emergency department (PED) from JulyNovember 2014 was conducted. Children were interviewed using a semi-structured format of ten open-ended questions. They were asked to describe their feelings when 1) they received something they wanted/ needed, 2) their expectations were met or not met in the ED, and 3) their pain was or was not relieved. A written survey was also completed by the caregiver. Interviews were transcribed and grounded theory was employed for data coding and analysis. Results: 105 child interviews were completed $(\mathrm{n}=53$ female, mean age 9.91 SD 3.71, age range 4-16). 105 caregiver surveys were completed ( $\mathrm{n}=80$ female). "Good", "better," and "happy" were most commonly used by all children $(\mathrm{n}=99)$ to express satisfaction with pain management $(27 \%, 21 \%$ and $22 \%$, respectively), with PED care $(31 \%, 14 \%$ and $33 \%)$ and in general $(13 \%, 5 \%$ and $49 \%)$. Children $(n=99)$ used the words "sad", "bad," and "not good" to communicate dissatisfaction with pain management (21\%, $7 \%$ and $11 \%$ respectively), and with PED care $(21 \%, 13 \%$ and $12 \%$, respectively). Only $55 \%$ of children understood the meaning of the word 'satisfaction'. Children used words that were similar to their caregiver $14 \%$ of the time. Conclusion: The word "satisfaction" should not be used to communicate with children in the emergency department, as many lack understanding of the term. The vocabulary that children use to describe satisfaction does not largely vary with context and involves simpler words than their parents. Caregiver vocabulary should not be used as a surrogate for pediatric patients. This study will inform the development of a validated tool to measure children's satisfaction with pain management.

Keywords: children, pain, satisfaction

\section{P007}

Association between serum biomarkers and frailty level in seniors with minor injurys

N. Allain-Boulé, MSc, J. Lebon, Ph.D, M. Sirois, PhD, M. Aubertin-Leheudre, PhD, M. Émond, MD, MSc; Centre de Recherche du CHU de Québec, Québec City, QC

Introduction: Frailty is associated with mobility \& physical impairment in seniors with minor injuries. Serum biomarkers have also been 
suggested as potential markers of these impairments in clinical studies. No study has examined if serum biomarkers could contribute to the diagnosis of frailty in seniors with minor injuries. Objectives: To explore the association between several serum biomarkers (Ferritin, Creatinine, Vitamin D, Albumin, Glucose, Estradiol, Testosterone, Thyroid Stimulating Hormone (TSH), Insulin-Growth Factor (IGF-1) C-reactive protein (CRP)) and frailty level in seniors treated in emergency department (ED) for minor injuries. Methods: Cross-sectional study within the larger CETI cohort. It includes 142 seniors discharged home from 4 EDs after treatment of minor injuries. Their frailty status was measured by the Canadian Study of Health \& Aging-Clinical Frailty Scale (CSHA-CFS). Biomarkers were obtained from blood samples. Pearson's correlations (r) were performed to examine the relation between serum biomarkers and frailty levels. Partial correlation controlled for age and sex, were also performed. Results: Due to inclusion criteria, no patient was severely frail. Overall, these preliminary analyses seem to indicate that robust patients tended to have lower Glucose \& Vitamin D levels $(-0.264 \leq \mathrm{r} \leq-0.230$; $\mathrm{p}<0.05)$ higher Estradiol $(r=0,230 ; p<0.05) \&$ Testosterone $(r=0,295$; $\mathrm{p}<0.05)$, while prefrail/frail patients tended to have higher Glucose \& Vitamin D levels $(0,235 \leq \mathrm{r} \leq 0,238 ; \mathrm{p}<0.05)$, lower Estradiol levels $(r=-0,235 ; p<0.05) \&$ more elevated Ferritin levels. Due to the small number of patients, controlling for age and sex lead to nonsignificant results of the most associations. Conclusion: Pre-frail/frail seniors presenting to EDs with minor injuries tend to have higher Ferritin, Glucose \& Vitamin D levels as well as lower sexual hormones levels than robust individuals. Larger samples are needed in order to elucidate which biomarkers could be most useful to identify frail seniors needing clinical attention and to assess a possible association with mobility impairments in this population.

Keywords: functional decline, biomarkers, frailty

\section{P008}

Addressing chronic pain and problematic substance use of opioids in the emergency department: can a comprehensive framework for care work?

M.A. Allen, MD; St. Martha's Regional Hospital, Antigonish, NS

Introduction: Chronic pain and substance misuse are complex chronic illness that are subject to prejudice, misinterpretation and cultural biases. They require a broad, multi-disciplined approach if they are to be effectively managed. Barrier's to effective care in the emergency department include our reliance on pain scales alone to effectively triage and manage chronic pain, differing philosophy's and attitudes regarding the appropriate use of high risk pain pharmacology including opioids and confusing classifications systems used to describe pain and problematic substance use which can often lead to further stigmatization and over medicating. Methods: The charts of thirteen patients with complex pain and problematic substance use who had more than 360 visits to a regional emergency department in one year were reviewed retrospectively and data collected regarding frequency of visits and disposition after the implementation of a comprehensive pain and addiction strategy from April 2011 to August 2015. Results: In the first year of implementation there was a $70 \%$ drop in the frequency of emergency department visits. Five patients $(38 \%)$ required a comprehensive pain plan. Six agreed $(46 \%)$ to a direct referral to our mental health, addiction and chronic pain services. Two patients (15\%) were lost to follow-up. Review of the electronic record to track patient visits to provincial emergency departments did not show an increase in visits to other facilities within the province. Review of visits in 2015 show a 97\% drop in the frequency of visits. Family Physicians in the community did not report an increase in the frequency of patient visits as a direct result of the implementation of this strategy nor was there an increase in complaints to administration regarding ineffective pain management. Conclusion: Although survey numbers are low it suggests that a framework for the management of complex pain and substance use disorders can be successfully implemented in the emergency department improving timely access to appropriate management and resources for patients based on best practice to address their complex needs.

Keywords: complex pain, substance use disorders, comprehensive care plan

\section{P009}

Violence against health care professionals in Karachi: results from a mixed methods study

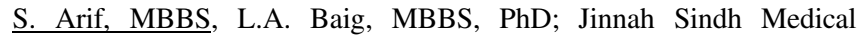
University, Karachi, Pakistan

Introduction: The right to live is the supreme human right and according to Article 3 of the Universal declaration of human rights everyone has a right to life, liberty and security. In Karachi, Pakistan huge numbers of health care professionals (HCP) have been subjected to violence inclusive of money extortion, kidnapping, mental \& physical torture, murders etc. A recent study from Karachi's four major hospitals reported that $72.5 \%$ of $\mathrm{HCP}$ have experienced abuse (verbal and physical) in the past 12 months. The goal of this study was to develop strategies for preventing violence against health care after collecting baseline data. The objectives of the study were to: Identify the magnitude, threshold and impact of violence against HCPs. Methods: This was a mixed methods study design with a QUAN-QUAL approach. Structured questionnaire was used after pilot testing and filled by the surveyors. Focus group discussion and In-depth interviews were conducted with HCPs, NGOs, Law enforcement agencies, ambulance services, hospital administrators and LHW programs. Frequencies and proportions were compared for different cadres of HCPs for the quantitative data analysis. Thematic content analysis with inductive and deductive reasoning was used for analysing qualitative data. Results: Data on 822 HCPs revealed that $33 \%$ had faced violence and $49 \%$ had experienced it, $89 \%$ was verbal and $43 \%$ was physical abuse, $2 \%$ had died and $22 \%$ were injured. Ambulance services and physicians were the most common victims of violence. The main reasons were grouped as institutional, behavioral (victims and perpetrators) and general situation of the city. There is high acceptance of violence among HCPs and lack of training in dealing with it was the most common reason given. Sequlae included effects on victim, family, institution and the reporting agencies. The final paper will have complete details on the baseline and the recommendations proposed by the stakeholders. Conclusion: Violence faced by HCP's is a multifactorial complex issue. There is a dire need to design interventions which can help in addressing the behavioral, Institutional and sociopolitical factors promoting violence among HCP's. The interventions based on recommendations by the respondents have been developed and implementation has started as a pilot in the city of Karachi.

Keywords: violence, health care professionals, ambulance services

\section{P010}

Use of the emergency department by refugees under the Interim Federal Health Program

F. Bakewell, MD, S. Addleman, MD, V. Thiruganasambandamoorthy, MS, MSc; University of Ottawa, Ottawa, ON 